TUM-HEP-469-02

MADPH-02-1283

hep-ph/0207133 v.2

October 29,2018

\title{
SUSY In The Sky: Observing Ultrahigh Energy Cosmic Neutralinos
}

\author{
Cyrille Barbot ${ }^{1}$, Manuel Drees ${ }^{1}$, Francis Halzen ${ }^{2}$ and Dan Hooper ${ }^{2}$ \\ ${ }^{1}$ Physik Dept., TU München, James Franck Str., D-85748 Garching, Germany \\ ${ }^{2}$ Department of Physics, University of Wisconsin, 1150 University Avenue, Madison, \\ WI 53706, USA
}

\begin{abstract}
In models where the ultra-high energy cosmic ray problem is solved by top-down scenarios, a significant flux of ultra-high energy neutralinos is predicted. We calculate the number of events expected from such particles in future experiments such as EUSO or OWL. We show that by using the Earth as a filter, showers generated by neutralinos can be separated from neutrino generated showers. We find that for many models, observable rates are expected.
\end{abstract}

\section{Introduction}

Cosmic ray observations have determined that the spectrum of the highest energy cosmic rays extends beyond $10^{20} \mathrm{eV}[1$. Observations have also indicated that the highest energy spectrum is dominated by protons rather than photons [2]. Above $\sim 5 \times 10^{19} \mathrm{eV}$, protons can interact with cosmic background photons at the $\Delta$-resonance generating pions. Above this energy, called the GZK (Greisen-Zatsepin-Kuzmin) cutoff [3], the proton energy loss length is near $50 \mathrm{Mpc}$, thus requiring semi-local sources to produce the observed flux. The lack of any such known sources has spawned a great deal of speculation as to the origin of these particles. A common class of models, called top-down scenarios, involve supermassive particles which decay or annihilate generating the highest energy cosmic rays [4, 5].

The decay of superheavy particles has been studied in some detail [6, 7, 8, 9, 10. In particular, it has been demonstrated that a significant amount of the initial energy of such a particle can be emitted in the form of ultra-high energy supersymmetric particles [6, 9, 10. In most models, the lightest supersymmetric particle is a neutralino. This neutralino, weakly interacting and stable by virtue of $R$-parity, can travel cosmological distances without absorption or scattering. In this paper, we discuss the prospects for observing ultra-high energy cosmic neutralinos in future very large area, satellite-borne air shower experiments.

\section{Ultra-High Energy Fragmentation To Neutralinos}

In the general framework of top-down scenarios, one has to consider the decay of superheavy $X$ particles with a mass of the order of $10^{21}$ to $10^{25} \mathrm{eV}$, and a lifetime comparable 
to or longer than the age of the universe. Such a long lifetime can be ensured by "storing" the $X$-particles in cosmological defects, which can survive into the present epoch [4, 11. Alternatively, free $X$-particles might be long-lived since their decay is suppressed, e.g. by (approximate) symmetries [12. For a review of different candidates, see [5]. Such particles could be produced in the very early times of the universe, e.g. at the end of inflation [13. The typical decay modes of the $X$ particles are generally unknown and/or quite model dependent. However, if there is no additional "new physics" scale between $M_{\mathrm{SUSY}} \sim 1 \mathrm{TeV}$ and $M_{X}{ }^{\dagger}$, the $X$ particles should decay into $N$ "known" particles of the minimal supersymmetric standard model (MSSM), and usual particle physics allows us to study in detail the shower generated by the primary products of the initial $X$ decay.

A detailed computation of the spectra of stable particles (protons, photons, neutralino LSPs, electrons and neutrinos of the three species) obtained in such decay showers has been described in [9, 15]. We recall here that at the energies we are considering, it is necessary to take into account all the gauge couplings of the MSSM; indeed, at the scale of unification, they are all of the same strength, so that electroweak (and some Yukawa) interactions can be as relevant as the QCD ones. The perturbative part of the shower was computed by solving numerically the complete set of evolution equations [15] for the relevant fragmentation functions of the MSSM. We carefully modeled the decays of unstable particles with mass near $M_{\mathrm{SUSY}} \sim 1 \mathrm{TeV}$, as well as the hadronization process at the GeV scale for light quarks and gluons. We found that the LSP flux depends only mildly on the spectrum of superparticles, as long as the LSP is a bino-like neutralino [15. Some sample spectra are shown in Fig. . $^{\ddagger}$

Here we have conservatively assumed that $X$ particles have an overdensity of $10^{5}$ in the vicinity of our galaxy, as expected [7] for $X$ particles that move freely under the influence of gravity. $§$ This minimizes the expected neutralino flux, since all scenarios are normalized by matching [16] the predicted proton spectrum at $E \simeq 10^{20} \mathrm{eV}$ to the highest energy cosmic ray observations. Data 2 indicate that most UHE events have protons (or heavier ions, which however cannot be produced in top-down models), not photons, as primary particles. Specifically, Yakutsk, Haverah Park and, most recently, AGASA observe more muons in the events than expected from photon primaries. The longitudinal development of the most energetic Fly's Eye event indicates that it is not due to a photon. Finally, the highest energy AGASA events should show a North-South asymmetry if they were due to photons (which, at that energy, initiate an electromagnetic cascade already in the Earth's magnetosphere), but no such asymmetry is observed. On the other hand, top-down models predict the photon flux at source to exceed the proton flux [15].

Following ref.[16] we are thus compelled to assume that most UHE photons are absorbed somewhere between source and Earth. According to ref. [5] this is in fact expected if most sources are at cosmological distances. At $E \simeq 10^{20} \mathrm{eV}$ the attenuation length for photons is at least one order of magnitude shorter than that for protons. This ratio is closer to 50, if the extragalactic $\vec{B}$ fields average at least $10^{-9} \mathrm{G}$, as is widely expected. On the other hand, according to standard estimates [17] the density of radio photons in our galaxy is only about

$\dagger$ This hypothesis, known as the "desert hypothesis", is well motivated by the fact that the existence of new physics between the GUT scale and the SUSY breaking scale would destroy the very impressive feature of "natural" unification of the gauge couplings at $M_{G U T} \sim 10^{16} \mathrm{GeV}$ occuring in the MSSM [14.

$\ddagger$ The primary 10-body decay $X \rightarrow 5 q 5 \tilde{q}$ has been modeled using phase space only, i.e. ignoring any possible dependence of the matrix element on external momenta.

$\S$ The exact profile of the halo of $X$ particles does not affect our results as long as most UHECR events originate at distances well below one GZK interaction length. 
one order of magnitude higher than the average value in intergalactic space, leading to an attenuation length of $10^{20} \mathrm{eV}$ photons of at least $100 \mathrm{kpc}$. In order to obtain near-complete absorption of such photons in our galaxy the actual density of radio photons would have to be at least ten times higher than the accepted value. This may not be impossible. The galactic (and extragalactic) radio background is reasonably well known only at frequencies above 100 $\mathrm{MHz}$. However, we are most interested in photons with frequency of only a few $\mathrm{MHz}$, which have the highest cross section for $e^{+} e^{-}$pair production with $10^{20} \mathrm{eV}$ photons. This frequency band is difficult to observe on Earth, due to strong absorption in the ionosphere.

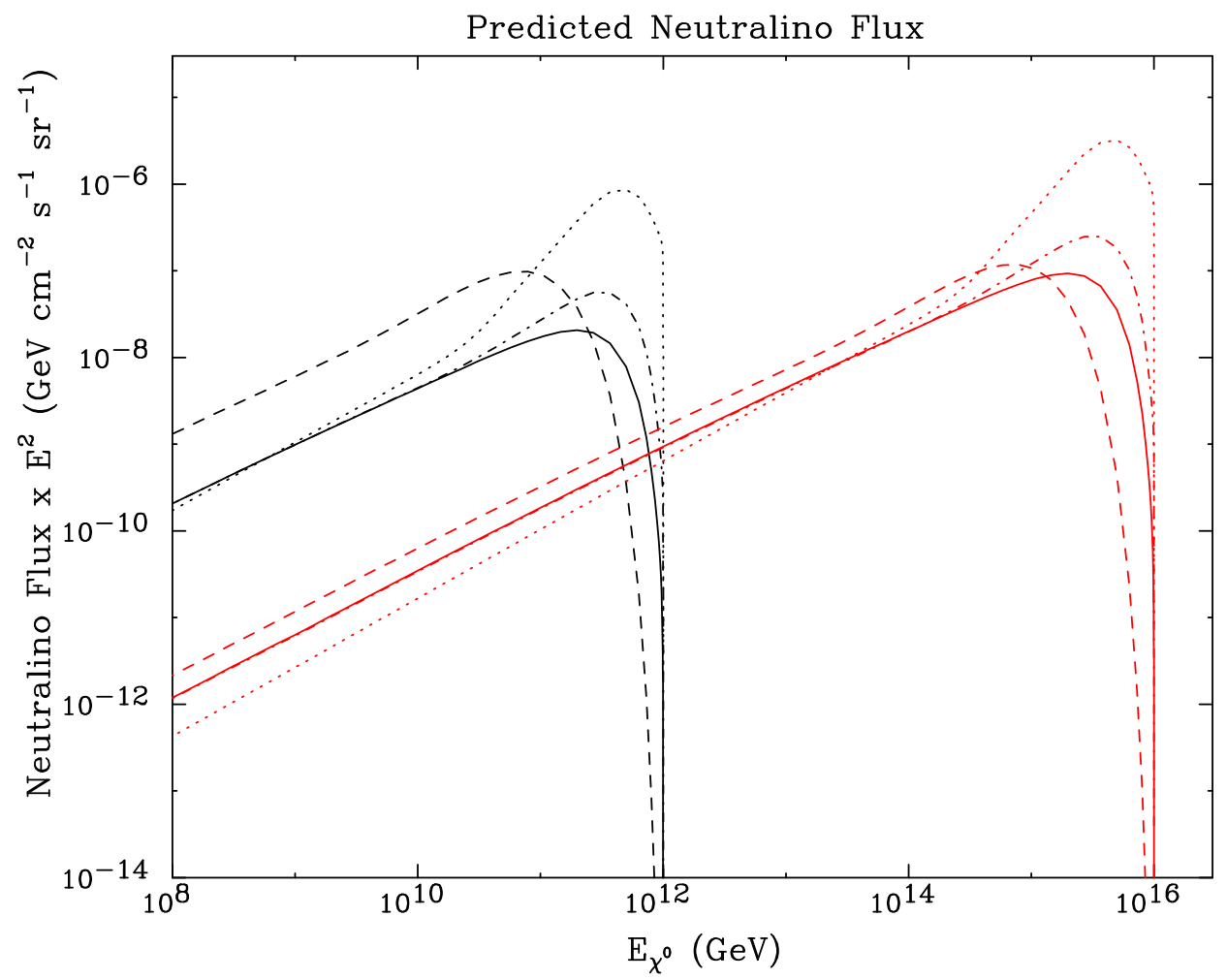

Figure 1: The spectrum of neutralino LSP's predicted for the decay of superheavy particles with mass $M_{X}=2 \cdot 10^{21} \mathrm{eV}$ (left set of curves) and $M_{X}=2 \cdot 10^{25} \mathrm{eV}$ (right) normalized [16] by the proton spectrum to the ultra-high energy cosmic ray flux, for a "galactic" distribution of sources where most UHECR events originate from $X$ decays in the halo of our galaxy. For a homogeneous distribution, the spectrum is enhanced by up to a factor of 15 . Spectra are shown for primary $X$ decays into quark+antiquark (solid), quark+squark (dot-dash), $S U(2)$ doublet lepton+slepton (dots) and 5 quark +5 squark (dashes). Note that for the case of $M_{X}=2 \cdot 10^{21} \mathrm{eV}$ decays, the spectrum peaks in the energy range most accessible to air shower experiments.

\section{Signatures of Ultra-High Energy Neutralinos}

Ultra-relativistic neutralinos interact with quarks by $t$-channel $Z$ and $W^{ \pm}$exchange, as well as by the exchange of squarks in the $s-$ or $u$-channel. These interactions either directly yield an LSP, or produce a heavier neutralino or chargino which quickly decays to the lightest neutralino 
(except, perhaps, in the case of near-degenerate masses). Either interaction generates a shower which can be observed by air shower experiments.

The background for this signal consists of showers generated by ultra-high energy cosmic neutrinos. The neutrino interaction length becomes comparable to the radius of the earth around $10^{5} \mathrm{GeV}$. By $10^{9} \mathrm{GeV}$, only about one out of 1000 neutrinos passes through the Earth without interaction (see figure 2). A neutralino, however, depending on the choice of SUSY parameters, will have a different interaction cross section and, therefore, different absorption properties. The size of this cross section depends sensitively on the neutralino eigenstate, which in general is a composition of bino, wino and neutral higgsinos. A wino- or higgsinolike neutralino has couplings to $W$ and/or $Z$ bosons that resemble or even exceed those of neutrinos. In contrast, a bino-like neutralino has very small couplings to gauge boson, because its superpartner, the $U(1)_{Y}$ gauge boson, does not couple to other gauge bosons. The couplings of bino-like neutralinos to squarks are of full $U(1)_{Y}$ gauge strength, but squark searches at the Tevatron [18 tell us that first and second generation squarks must be at least three times heavier than $W$ bosons. Note also that models with radiative breaking of the electroweak gauge symmetry prefer the lightest neutralino to be bino-like in most of parameter space 19. Typical parameter choices therefore predict neutralino-nucleon cross sections one or two orders of magnitude smaller than neutrino-nucleon cross sections [6]. With a significantly smaller cross section, very high energy cosmic neutralinos may travel through the Earth producing upgoing events at much higher energies than neutrinos. Upgoing showers with energy above $100 \mathrm{PeV}$ or so would be a smoking gun for cosmic neutralinos.

Furthermore, by virtue of $R$-parity, neutralinos will generate less energetic neutralinos in each interaction, thus not depleting their number. Tau neutrinos also display this property [20, but not as dramatically. The difference comes from the fact that high energy tau leptons lose energy in propagation whereas charginos decay quickly enough to lose very little energy in propagation. Also, phase space arguments indicate that a larger fraction of a decaying chargino's energy goes into the resulting (massive) neutralinos than a decaying tau's energy goes into the new (essentially massless) tau neutrino. Together, these effects indicate that tau regeneration is largely ineffective above about $10^{8} \mathrm{GeV}$. On the other hand, for even moderately smaller neutralino cross sections, the Earth can remain effectively transparent to cosmic neutralinos at much higher energies.

Our calculations of tau neutrino and neutralino regeneration in the Earth were done with a Monte Carlo simulation which, at each interaction, calculated the energy lost in the interaction and following propagation [20]. Our treatment of $\tau$ propagation includes $e^{+} e^{-}$pair production, photonuclear interactions, bremsstrahlung and ionization energy losses. As stated earlier, any unstable superparticle produced in LSP interactions is too short-lived to lose energy prior to its decay. We estimate that each interaction, if necessary followed by superparticle decay, will reduce the energy of the LSP by slightly more than a factor of two; this effect is included in our treatment of LSP regeneration. Our code demonstrated the appearance of a 'pile-up' of outgoing particles at an energy corresponding to an interaction length equal to the size of the Earth. For tau neutrinos, this occurs at PeV energies, but can be considerably higher for neutralinos, due to their smaller cross section. 


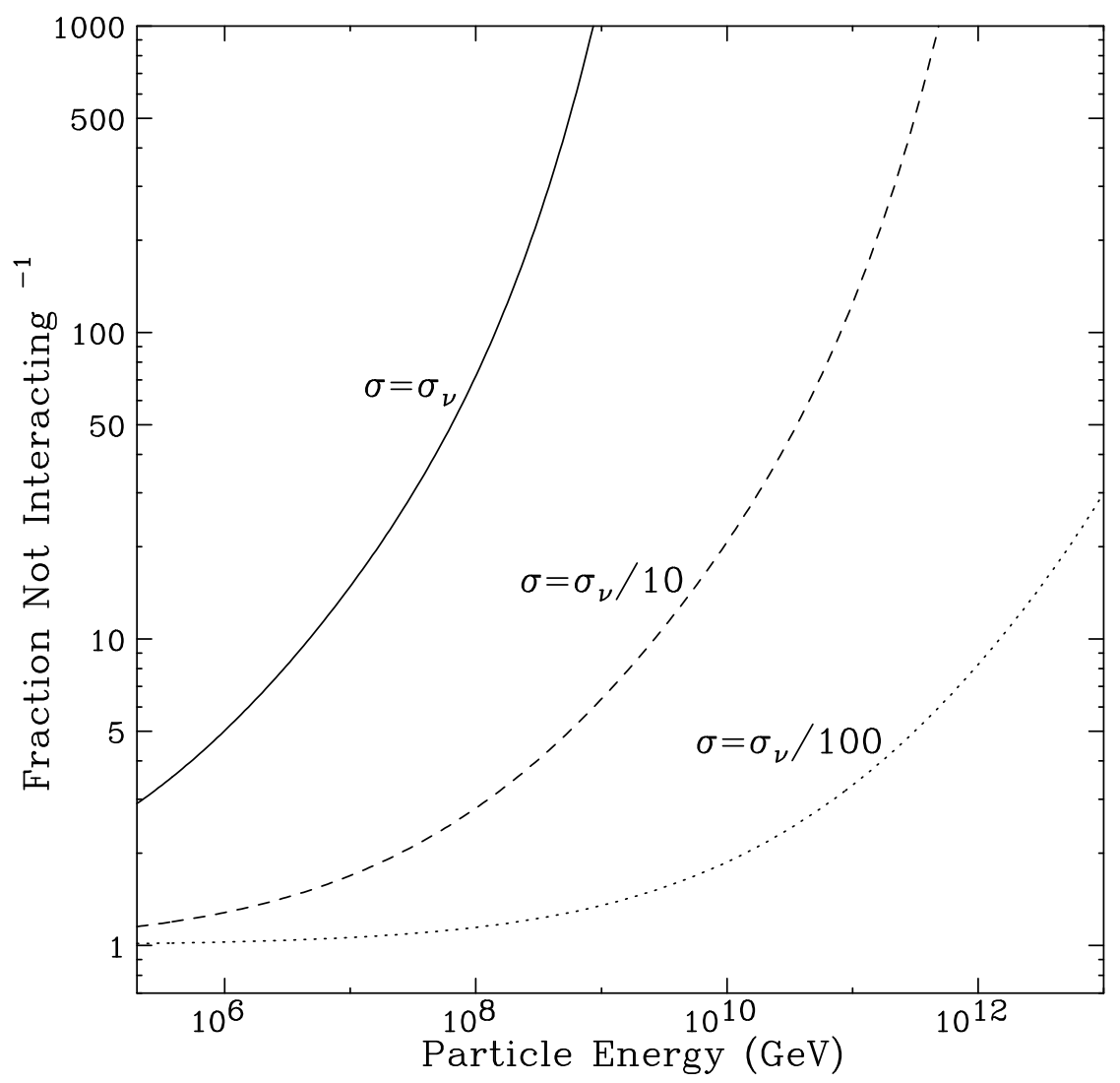

Figure 2: The fraction of neutrinos or neutralinos which pass through the Earth (integrated over zenith angle less than 85 degrees) as a function of energy. Results are shown for particles with total cross sections with nucleons equal to that for neutrinos as well as for particles with cross sections ten and one hundred times smaller. Regeneration effects are not included (see end of sec. 3).

\section{Prospects For Detection In Air Shower Experiments}

The flux of very high energy neutralinos from top-down scenarios can be calculated assuming that this is the mechanism which generates the highest energy cosmic rays [4, 8, 9, 15. Given a sufficient cosmic flux, these neutralinos may be detected in future air shower experiments. The challenge, however, is not merely observing the showers generated in neutralino interactions but in differentiating these cosmic neutralinos from neutrinos.

We have calculated the number of neutralino events predicted for a variety of top-down models associated with the highest energy cosmic rays in a future experiment such as EUSO 21] or OWL 22]. EUSO and OWL are proposed satellite experiments which observe fluorescence in the Earth's atmosphere generated in very high energy showers. Such experiments are expected to observe on the order of 150,000 square kilometers of surface area on the Earth. Particles which pass through the Earth can interact in the shallow Earth or atmosphere generating upgoing showers observable by fluorescence or Cerenkov radiation. Ultra-high energy showers reach a maximum near a slant depth of $850 \mathrm{~g} / \mathrm{cm}^{2}$, corresponding to a depth of 8.5 meters in water. Including the effective slant depth of the lower atmosphere extends this to $\sim 0.015 \mathrm{~km}$, thus providing a water equivalent effective volume of $\sim 150,000 \times 0.015 \sim 2250$ cubic kilometers, a truly enormous volume. Such an experiment will be capable of measuring 
both the energy and the direction of an observed particle.

Estimating the rate of neutrino-induced "background" events is difficult at present since the neutrino flux at $E \gtrsim 10^{9} \mathrm{GeV}$ is not known. The flux of atmospheric neutrinos is completely negligible at these energies. However, most proposed explanations of the UHECR events also predict a significant UHE neutrino flux. We therefore use the neutrino flux predicted by top-down models [16] to estimate the neutrino background. Fig. 3 compares signal and background at $E \geq 1 \mathrm{EeV}$ for one such model, where we assume a galactic distribution of $X$ particles, with primary $X \rightarrow q \bar{q}$ decay and $M_{X}=2 \cdot 10^{12} \mathrm{GeV}$. We see that signal and background clearly have very different angular distributions even for the larger LSP-nucleon cross section of $\sigma_{\nu} / 10$. Regeneration effects are included, but they cannot produce neutrino events at large energy and large angle. Requiring the events to emerge more than $5^{\circ}$ below the horizon removes almost all the background, with little loss of signal; in the case at hand, we expect about 2 signal events per year, compared to 0.1 background event. If the LSP-nucleon cross section is smaller, a somewhat stronger angular cut may be advantageous; on the other hand, at even higher energies it might be better to use a slightly weaker cut. However, this variation of the angular cut has negligible effect on the predicted signal rate, compared to the uncertainty inherent in our estimates. In the following we therefore apply a fixed angular cut of $5^{\circ}$ on the signal in all cases. This cut will have to be optimized once the angular resolution of the experiment is known. Moreover, measurements at neutrino telescopes as well as AUGER should soon greatly improve our knowledge of the neutrino flux at very high energies. Finally, this figure also shows that a measurement of the angular distribution of the signal will allow to determine the LSP scattering cross section: for the larger cross section shown, there will be very few vertically upgoing events. The dependence of the angular distribution of the signal on the cross section becomes even more pronounced at higher energies.

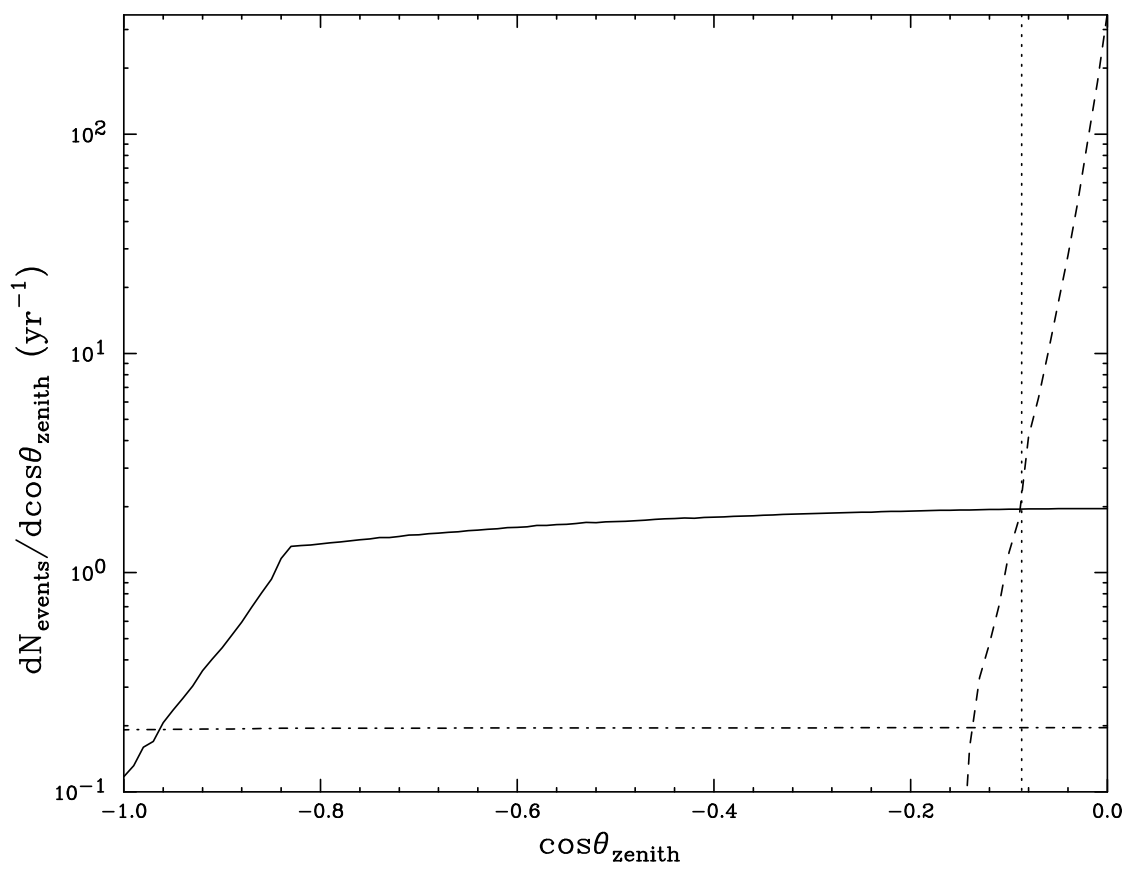

Figure 3: The neutrino background (dashed) and LSP signal (solid: $\sigma_{\mathrm{LSP}}=\sigma_{\nu} / 10$; dot-dashed: $\left.\sigma_{\mathrm{LSP}}=\sigma_{\nu} / 100\right)$ at $E>1 \mathrm{EeV}$. Both signal and background result from $X \rightarrow q \bar{q}$ decays of $2 \cdot 10^{12} \mathrm{GeV} X$ particles with a galactic distribution. The vertical dotted line indicates the angular cut of $5^{\circ}$ applied to the signals listed in table 1. 


\begin{tabular}{|c||c|c|}
\hline \hline$E_{\chi^{0}} \geq 1 \mathrm{EeV}$ & $\sigma_{\chi^{0}}=\sigma_{\nu} / 10$ & $\sigma_{\chi^{0}}=\sigma_{\nu} / 100$ \\
\hline \hline$q \bar{q}, 10^{21} \mathrm{eV}$, Galactic & 1.86 & 0.196 \\
$q \tilde{q}, 10^{21} \mathrm{eV}$, Galactic & 2.96 & 0.306 \\
$5 \times q \tilde{q}, 10^{21} \mathrm{eV}$, Galactic & 4.05 & 0.436 \\
$l \tilde{l}, 10^{21} \mathrm{eV}$, Galactic & 28.0 & 2.81 \\
\hline \hline$q \bar{q}, 10^{25} \mathrm{eV}$, Galactic & 0.187 & 0.0189 \\
$q \tilde{q}, 10^{25} \mathrm{eV}$, Galactic & 0.213 & 0.0216 \\
$5 \times q \tilde{q}, 10^{25} \mathrm{eV}$, Galactic & 0.213 & 0.0216 \\
$l \tilde{l}, 10^{25} \mathrm{eV}$, Galactic & 0.615 & 0.0617 \\
\hline \hline$q \bar{q}, 10^{21} \mathrm{eV}$, Homogeneous & 27.9 & 2.94 \\
$q \tilde{q}, 10^{21} \mathrm{eV}$, Homogeneous & 44.4 & 4.56 \\
$5 \times q \tilde{q}, 10^{21} \mathrm{eV}$, Homogeneous & 60.8 & 6.54 \\
$l \tilde{l}, 10^{21} \mathrm{eV}$, Homogeneous & 420.0 & 42.15 \\
\hline \hline$q \bar{q}, 10^{25} \mathrm{eV}$, Homogeneous & 2.81 & 0.284 \\
$q \tilde{q}, 10^{25} \mathrm{eV}$, Homogeneous & 3.20 & 0.324 \\
$5 \times q \tilde{q}, 10^{25} \mathrm{eV}$, Homogeneous & 3.20 & 0.324 \\
$l \tilde{l}, 10^{25} \mathrm{eV}$, Homogeneous & 9.23 & 0.926 \\
\hline \hline \hline$E_{\chi} \geq 100$ EeV & $\sigma_{\chi^{0}}=\sigma_{\nu} / 10$ & $\sigma_{\chi^{0}}=\sigma_{\nu} / 100$ \\
\hline \hline$q \bar{q}, 10^{21} \mathrm{eV}$, Galactic & 0.0976 & 0.0344 \\
$q \tilde{q}, 10^{21} \mathrm{eV}$, Galactic & 0.391 & 0.122 \\
$5 \times q \tilde{q}, 10^{21}$ eV, Galactic & 0.0161 & 0.00716 \\
$l \tilde{l}, 10^{21} \mathrm{eV}$, Galactic & 10.1 & 2.38 \\
\hline \hline$q \bar{q}, 10^{25} \mathrm{eV}$, Galactic & 0.0946 & 0.0143 \\
$q \tilde{q}, 10^{25} \mathrm{eV}$, Galactic & 0.116 & 0.0169 \\
$5 \times q \tilde{q}, 10^{25} \mathrm{eV}$, Galactic & 0.103 & 0.0159 \\
$l \tilde{l}, 10^{25} \mathrm{eV}$, Galactic & 0.435 & 0.0576 \\
\hline \hline$q \bar{q}, 10^{21} \mathrm{eV}$, Homogeneous & 1.46 & 0.516 \\
$q \tilde{q}, 10^{21} \mathrm{eV}$, Homogeneous & 5.87 & 1.83 \\
$l \tilde{q}, 10^{21} \mathrm{eV}$, Homogeneous & 0.242 & 0.107 \\
$q \bar{q}, 10^{25} \mathrm{eV}$, Homogeneous & 151.5 & 35.7 \\
\hline \hline $1 \tilde{q}, 10^{25} \mathrm{eV}$, Homogeneous & 1.74 & 0.215 \\
$l \tilde{q}, 10^{25} \mathrm{eV}$, Homogeneous & 1.55 & 0.254 \\
\hline \hline
\end{tabular}

Table 1: Neutralino event rates per year in top-down scenarios in a large area air shower experiment such as EUSO or OWL, with effective volume $\simeq 2250$ cubic kilometers (water equivalent). Rates are shown for two choices of neutralino-nucleon cross sections, two choices of energy threshold and several top-down models. At the energies considered, there is very little neutrino background for upgoing events (see text).

Table 1 shows signal event rates for two choices of energy threshold, $E_{\chi^{0}} \geq 1 \mathrm{EeV}$ and 100 $\mathrm{EeV}$. We also show results for the stronger cut on energy in order to illustrate that at least in some cases the LSP spectrum should be measurable over a significant range of energies. The first case shown in the table corresponds to the situation depicted in Fig. 3. Of course, the choice of a $100 \mathrm{EeV}$ threshold is even more effective in reducing the background, to the level of $10^{-3}$ events per year. From the physics point of view an energy threshold of 100 EeV should only be necessary in the unlikely case that the total background of ultra-high energy neutrinos is dominated by some mechanism not related to the observed UHECR events. Regarding the energy threshold which can be achieved experimentally, it has been argued that for upgoing 
events, the threshold could be as small as a PeV [23].

The rates shown in table 1 are for a variety of primary $X$ decay modes, and for "galactic" and homogeneous distributions of $X$ particles. It seems highly unlikely that $X$ particles will indeed be distributed homogeneously, but it is conceivable that the majority of sources contributing to the LSP flux is at cosmological distances (e.g. if the $X$ particles are embedded in topological defects); the homogeneous distribution is meant to be representative for such models. Our results show that the $X$ distribution throughout the universe has significant impact on the expected size of our signal. For a full description of these models, see our previous paper [16]. We note that the neutralino signal is more sensitive to the primary $X$ decay mode than the neutrino signal analyzed in [16] is. Not surprisingly, scenarios with (at least) one superparticle in the primary decay produce a higher neutralino flux than models where $X$ only decays into quarks. Moreover, leptonic $X$ decays increase the predicted neutralino flux by another order of magnitude, since in this case relatively few protons are produced, leading to a higher source density required to explain the observed UHECR events. On the other hand, choosing $M_{X}=2 \cdot 10^{25} \mathrm{eV}$ rather than $2 \cdot 10^{21} \mathrm{eV}$ significantly reduces the predicted flux. Note, however, that in this case $X$ decays can only describe the UHECR flux above $\sim 10^{20} \mathrm{eV}$ [16]; events at a few times $10^{19} \mathrm{eV}$ then have to be produced by an as yet unknown source.

As stated earlier, we normalize the LSP flux by assuming that (almost) all UHE photons are absorbed between source and Earth, as indicated by experiment 2]. Since absorption of UHE photons in our galaxy is speculative, we comment on how the expected signal is changed if this evidence is ignored, i.e. if the observe UHECR spectrum is normalized to the sum of photon and proton fluxes. The predicted LSP event rate for galactic models with $M_{X}=2 \cdot 10^{25}$ $\mathrm{eV}$ would go down by about a factor of 4 . If $M_{X}=2 \cdot 10^{21} \mathrm{eV}$, the predicted event rate would go down by a factor of 2 to 3 for hadronic primary $X$ decays, and by about an order of magnitude for purely leptonic primary $X$ decay. " Note that this "uncertainty" in the predicted event rate from taking refs. 2] seriously or not is comparable to the variation between different primary $X$ decay modes. Finally, we remind the reader that the UHECR spectra measured by AGASA and HiRes differ significantly in the post-GZK region, where we normalize our fluxes, leading to a corresponding uncertainty in our predicted signal.

\section{Conclusions}

The cosmic neutralino flux predicted in top-down scenarios could possibly provide an interesting test of both supersymmetry and GUT scale particle physics. To identify any showers generated in future experiments as being generated by cosmic neutralinos, they will need to occur at energies and from directions at which neutrinos would be absorbed by the Earth. We have calculated the event rates for a variety of such models for a large area air shower experiment such as OWL or EUSO. We find that for many scenarios, the event rate is large enough to be observable in principle. We should mention here that our estimates of annual event rates assume $100 \%$ duty cycle. This is clearly not realistic for any experiment based on optical observations. However, planning for the kind of space-based experiment we envision is still in its early stage; a smaller duty cycle might be compensated by a larger area and/or a longer period of observation.

\footnotetext{
TThe UHE neutrino background from $X$ decay would be reduced by the same factor.
} 
We believe that searching for UHE LSPs is very important, since it is the only measurement that can qualitatively distinguish between "top-down" and the more conventional "bottomup" explanations for the observed UHE events: in bottom-up models superparticles can only be produced in the collision of accelerated protons, so the UHE LSP flux will be a tiny fraction [typically $\mathcal{O}\left(10^{-6}\right)$ or less] of the UHE neutrino flux, much too small to be observed in any currently conceivable experiment. In contrast, a sizable UHE LSP flux is a generic prediction of top-down models. Moreover, the neutralino event rate turns out to be a far more sensitive probe of details of the model than the flux of neutrinos with energy exceeding $\sim 1 \mathrm{PeV}[16$. We therefore find it encouraging that the observation of UHE LSPs along the lines suggested in this paper, while certainly not easy, should at least be possible.

Acknowledgments: We thank Andrew Strong for a useful discussion. This work was supported in part by a DOE grant No. DE-FG02-95ER40896 and in part by the Wisconsin Alumni Research Foundation. The work of M.D. was partially supported by the SFB375 of the Deutsche Forschungsgemeinschaft.

\section{References}

[1] M. Takeda et al., AGASA collab., Phys. Rev. Lett. 81, 1163 (1998), astro-ph/9807193; T. Abu-Zayyad et al., HiRes collab., astro-ph/0208301.

[2] R. A. Vazquez et al., Astroparticle Physics 3, 151 (1995); M. Ave et al., Phys. Rev. Lett. 85, 2244 (2000), astro-ph/0007386, and Phys. Rev. D65, 063007 (2002), astro-ph/0110613 K. Shinozaki et al., AGASA collab., Astrophys. J. 571, L120 (2002).

[3] K. Greisen, Phys. Rev. Lett. 16, 748 (1966); G. T. Zatsepin and V. A. Kuzmin, JETP Lett. 4, 78 (1966) [Pisma Zh. Eksp. Teor. Fiz. 4, 114 (1966)].

[4] C.T. Hill, D. N. Schramm and T.P. Walker, Phys. Rev. D36, 1007 (1987); P. Bhattacharjee, C. T. Hill and D. N. Schramm, Phys. Rev. Lett. 69, 567 (1992).

[5] For a review, see P. Bhattacharjee and G. Sigl, Phys. Rep. 327, 109 (2000).

[6] V. Berezinsky and M. Kachelriess, Phys. Lett. B422, 163 (1998), hep-ph/9709485.

[7] M. Birkel and S. Sarkar, Astropart. Phys. 9, 297 (1998), hep-ph/9804285.

[8] V. Berezinsky and M. Kachelriess, Phys. Rev. D63, 034007 (2001), hep-ph/0009053; Z. Fodor and S.D. Katz, Phys. Rev. Lett. 86, 3224 (2001), hep-ph/0008204 C. Coriano and A.E. Faraggi, Phys. Rev. D65, 075001 (2002), hep-ph/0106326 S. Sarkar and R. Toldra, Nucl. Phys. B621, 495 (2002), hep-ph/0108098.

[9] C. Barbot and M. Drees, Phys. Lett. B533, 107 (2002), hep-ph/0202072.

[10] A. Ibarra and R. Toldra, JHEP 0206, 006 (2002), hep-ph/0202111.

[11] V. Berezinsky and A. Vilenkin, Phys. Rev. Lett. 79, 5202 (1997), astro-ph/9704257. 
[12] J.R. Ellis, J.L. Lopez, D.V. Nanopoulos, Phys. Lett. B247, 257 (1990); K. Benakli, J.R. Ellis and D.V. Nanopoulos, Phys. Rev. D59, 047301 (1999), hep-ph/9803333; K. Hamaguchi, Y. Nomura and T. Yanagida, Phys. Rev. D58, 103503 (1998), hep-ph/9805346, and Phys. Rev. D59, 063507 (1999), hep-ph/9809426, K. Hamaguchi, K.I. Izawa, Y. Nomura and T. Yanagida, Phys. Rev. D60, 125009 (1999), hep-ph/9903207; K. Hagiwara and Y. Uehara, Phys. Lett. B517, 383 (2001), hep-ph/0106320 C. Coriano, A. E. Faraggi and M. Plümacher, Nucl. Phys. B614, 233 (2001), hep-ph/0107053.

[13] D.J.H. Chung, E.W. Kolb and A. Riotto, Phys. Rev. Lett. 81, 4048 (1998), hep-ph/9805473 D.J.H. Chung, E.W. Kolb, A. Riotto and I.I. Tkachev, Phys. Rev. D62, 043508 (2000), hep-ph/9910437; D.J.H. Chung, P. Crotty, E.W. Kolb and A. Riotto, Phys. Rev. D64, 043503 (2001), hep-ph/0104100, R. Allahverdi and M. Drees, Phys. Rev. Lett. 89, 091302 (2002), hep-ph/0203118], and Phys. Rev. D66, 063513 (2002), hep-ph/0205246.

[14] U. Amaldi, W. de Boer and H. Fürstenau, Phys. Lett. B260 (1991) 447; P. Langacker and M. Luo, Phys. Rev. D44 (1991) 817; J. Ellis, S. Kelley and D.V. Nanopoulos, Phys. Lett. B260 (1991) 131; C. Giunti, C.W. Kim and U.W. Lee, Mod. Phys. Lett. A6 (1991) 1745 .

[15] C. Barbot and M. Drees, hep-ph/0211406, to be published in Astroparticle Physics.

[16] C. Barbot, M. Drees, F. Halzen and D. Hooper, Phys. Lett. B555, 22 (2003), hep-ph/0205230.

[17] A.W. Stong, I.V. Moskalenko and O. Reimer, Astrophys. J. 537, 763 (2000), Erratum: 541, 1109 (2000), astro-ph/9811296.

[18] D0 collab., S. Abachi et al., Phys. Rev. Lett. 75, 618 (1995); CDF collab., F. Abe et al., Phys. Rev. D56, 1357 (1997).

[19] See e.g. B.C. Allanach et al., hep-ph/0202233, presented at APS/DPF/DPB Summer Study on the Future of Particle Physics (Snowmass 2001), Snowmass, Colorado, 30 June - 21 July 2001.

[20] F. Halzen and D. Saltzberg, Phys. Rev. Lett. 81, 4305 (1998), hep-ph/9804354; J. F. Beacom, P. Crotty and E. W. Kolb, Phys. Rev. D66, 021302 (2002), astro-ph/0111482; S. I. Dutta, M. H. Reno and I. Sarcevic, Phys. Rev. D62, 123001 (2000), hep-ph/0005310.

[21] L. Scarsi, in Metepec 2000, Observing ultrahigh energy cosmic rays from space and earth 113-12\%; O. Catalano, Nuovo Cim. 24C, 445 (2001).

[22] D. B. Cline, prepared for the Ultra High-Energy Cosmic Ray Workshop on Observing Giant Cosmic Ray Air Showers for $>10^{20}$ eV Particles from Space, College Park, MD, 13-15 Nov 1997; R. E. Streitmatter [OWL Collaboration], prepared for the Ultra HighEnergy Cosmic Ray Workshop on Observing Giant Cosmic Ray Air Showers for $>10^{20}$ eV Particles from Space, College Park, MD, 13-15 Nov 1997.

[23] D. B. Cline and F. W. Stecker, astro-ph/0003459. 